\title{
Dietary and Life Habits of Obesity and Brown Rice Eaters among Genmai Evidence for Nutritional Kenko Innovation (GENKI) Study I and II
}

\section{Masahito Takahashi ${ }^{1}$, Shaw Watanabe ${ }^{2 *}$, Azusa Hirakawa ${ }^{3}$ and Shoich Mizuno $^{4}$}

${ }^{1}$ Department of Life Sciences, Graduate School of Arts and Sciences, The

University of Tokyo, Japan

${ }^{2}$ Lifescience Promoting Association, Japan

${ }^{3}$ Asian Nutrition and Food Culture Research Center, Japan

${ }^{4}$ National Cancer Center East, Japan

*Corresponding Author: Shaw Watanabe, Lifescience Promoting Association, Tokyo, Japan.
Received: June 29, 2020

Published: July 16, 2020

(c) All rights are reserved by Shaw

Watanabe., et al.

DOI: $10.31080 /$ ASNH.2020.04.0730

\section{Abstract}

The relationship between diet and health is a field that requires more research in an ultra-aging society. A questionnaire survey was conducted, to compare groups of brown rice and natural foods eaters, with residential population to clarify the relationship between the brown rice eaters and obese people. The total number of respondents was 7183 . The odds ratio to be obesity was 1.67 among white rice eaters, while it was 0.48 among brown rice eaters.

The consumption of food items as side dishes showed a characteristic trend among white rice eaters, while it was 0.48 among brawn rice eaters. Brown rice eaters consumed significantly more carrots, green yellow vegetables, burdock, lotus roots, pumpkin, sweet potatoes, yam, sesame, salty plum pickles (umeboshi), peanut, chestnut, mushrooms, dried mushrooms (shiitake), sea weed (nori, konbu), red bean (azuki), and soy milk. They did not consume meat and fish, but soy protein and other plant protein substituted to meat and fish.

The polished white rice eaters of obesity group preferentially consumed sweet bread, broccoli, bell pepper, eggplant, banana, grapefruit, red meat fish like tuna, beaf, cow and pig meat, egg, dairy products, sugar, and mayonnaise. They also consumed coffee and soft drinks, chocolate, cakes, ice cream and jelly.

Brown rice contains many functional ingredients that have various effects on physiological functions, such as innate immunity, recognition, etc., so these should be effective in aging and post corona society.

Keywords: Brown Rice; Obesity; Dietary Habits; Life Habits; Functional Ingredient

\section{Introduction}

The obesity has been the main cause of type 2 diabetes in the world, but the Asian risk in developing diabetes mellitus caused by obesity was several times higher than that of Westerners [1]. The incidence rate of diabetes mellitus among Westerners was only $7-8 \%$ at BMI $30 \mathrm{~kg} / \mathrm{m}^{2}$, but it reached about $25 \%$ among Japanese at the same BMI. Furthermore, the insulin secretory ability declined with aging, then diabetes patients became a national disease like cancer in the aging society [2].
After implementation of "Healthy Japan21" as an approach to prevent obesity by diet and physical activity, the number of patients with metabolic syndrome remained flat. But the number of patients with diabetes continued to increase and reached almost 10 million now in Japan [3-6]. Therefore, it is the most important to decrease the number of patients with diabetes and its complication by preventing obesity [5-10]. 
We have studied to substantiate the health promotion effect of brown rice by the Genmai Evidence for Nutritional Genki Innovation (GENKI) Study 1 and 2 [11,12] and investigated in detail of the daily life, eating habits and particularly about food of obese people, and analyzed it in comparison with the self-rating of health status and the degree of vitality of brown rice eaters by cohort studies of 7183 participants. This study focused on the eating habits of obese people in comparison with brown rice eaters by combining GENKI1 and GENKI2 Study participants.

\section{Ethical issue}

The current study protocol has been reviewed and approved by the Ethics Review Board of the Life Science Promoting Association (\#002-2016). Signed agreement to participate in the study was collected at the time of first questionnaire survey. Each participant's data were given an ID number for statistical analysis, and it was made anonymous to protect privacy.

\section{Methods}

Recruiting participants for the study was announced in a local magazine or pamphlets at their workplace, mostly macrobiotic vegetarians participated more than 1200 people, in "GENKI Study I" (hereinafter, this is called "GENKI I"). A total 5983 people have participated in the survey of "GENKI Study II" (hereinafter, this is called "GENKI II") from more than 30 workplaces concerning food or agriculture. The participants were staff members and their family members from Rice companies (19\%), Agricultural Cooperative Associations (46\%), restaurants, nutrition schools, etc., who lived in different areas of Japan.

GENKI I and II recruited participants of both men and women aged 20 - 79 and the questionnaire comprehensively asked about the past medical history, current symptoms, what kind of rice to eat as staple food, eating habits, taste for food, ingestion state of food, lifestyle, etc. The questionnaire was open on http://lifescience. or.jp. We created the Excel database and analyzed in IBM-SPSS ver. 24. For easy comparison, the odds ratio (OR) of obese group and non-obese group, and in brown rice group OR against non-brown rice group was shown for each factor in the tables 1-4.

\section{Results}

Obese people in the participants with BMI more than $25 \mathrm{~kg} / \mathrm{m}^{2}$ or higher was 1243 out of 7169 . The obese people were already pre-obese (OR 8.74) or Obese-class I (OR 46.76) when they had been 20 years old, so these were a risk of obesity in later life.
As for the kind of staple food for obese people were mostly polished white rice and the odds ratio (OR) to be obese was 1.68, in contrast with that brown rice was less than half (OR 0.45) (Table 1).

\begin{tabular}{|l|c|c|c|c|c|c|c|}
\hline & Type of rice & Total & Obesity & \% & OR & p & \\
\hline 1 & Polished white rice & 4774 & 922 & $19 \%$ & 1.68 & 0.00 & $* * *$ \\
\hline 2 & Half-polished rice & 376 & 49 & $13 \%$ & 0.69 & 0.02 & $*$ \\
\hline 3 & Haiga-mai (rice bud rich) & 122 & 22 & $18 \%$ & 1.12 & 0.64 & \\
\hline 4 & Brown rice & 777 & 67 & $9 \%$ & 0.45 & 0.00 & $* * *$ \\
\hline 5 & $\begin{array}{c}\text { Mixture of 16 different } \\
\text { kinds of cereals }\end{array}$ & 306 & 45 & $15 \%$ & 0.99 & 0.97 & \\
\hline 6 & $\begin{array}{c}\text { Kinmemai rice (Partial } \\
\text { removal of bran layer) }\end{array}$ & 579 & 97 & $17 \%$ & 0.94 & 0.58 & \\
\hline 7 & Wax free brown rice & 235 & 41 & $17 \%$ & 0.92 & 0.65 & \\
\hline
\end{tabular}

Table 1: Rice and risk of obesity.

The answer of current health conditions in obese people were mostly "Not healthy" (OR 1.71) and "Not very healthy" (OR 1.84) and the answer to "Very healthy" was low (OR 0.51). On the contrary, in the answer in brown rice eaters "Not healthy" were less, (OR 0.87) and "Healthy" (OR 1.79) was most frequent. This trend was also seen in the answer of "Health status compared to one year before", and many obese people answered "Worse" (OR 1.41), few were "Better" (OR 0.60), that was opposite results in brown rice eaters.

The past medical history of diseases in obese people showed significantly high risk in many lifestyle related diseases, such as diabetes (OR 2.36), hypertension (OR 2.98), asthma (OR 1.33), bronchitis (OR 2.72), and gallstones (OR 1.88). Current prescribed drug use showed also high OR for many lifestyle related diseases among obese people, while brown rice eaters showed almost one tenth $\mathrm{OR}$ in hypertension, angina, and hyperlipidemia (Table 2).

The past medical history showed higher odds ratios for allergies (OR 1.45) and cystitis (OR 1.61) in brown rice eaters. The anti-allergic effects of brown rice had been well known, so dietary change to brown rice could be happen, expecting an improvement of the diseases.

Regarding a stress, many obese people answered "Strong" (OR 1.40 ), but many of the brown rice eaters answered "Not so much" (OR 1.69), and significantly fewer "Strong" (OR 0.81). Only a small 


\begin{tabular}{|c|c|c|c|c|c|c|c|c|c|c|c|c|}
\hline & & & \multicolumn{5}{|c|}{ Obesity Group } & \multicolumn{5}{|c|}{ Brown rice eater Group } \\
\hline \multicolumn{13}{|c|}{$\begin{array}{l}\text { Have you ever been told by a doctor that } \\
\text { you have the following illness? }\end{array}$} \\
\hline 1 & No & 3117 & 460 & $14.8 \%$ & 0.69 & 0.000 & $* * *$ & 366 & $11.7 \%$ & 0.88 & 0.107 & \\
\hline 2 & Diabetes Mellitus & 226 & 88 & $38.9 \%$ & 2.36 & 0.000 & $* * *$ & 31 & $13.7 \%$ & 0.86 & 0.475 & \\
\hline 4 & Hypertension & 750 & 275 & $36.7 \%$ & 2.98 & 0.000 & $* * *$ & 80 & $10.7 \%$ & 0.48 & 0.000 & *** \\
\hline 5 & Angina & 71 & 23 & $32.4 \%$ & 1.52 & 0.127 & & 6 & $8.5 \%$ & 0.44 & 0.062 & \\
\hline 6 & Asthma & 353 & 75 & $21.2 \%$ & 1.33 & 0.045 & $*$ & 52 & $14.7 \%$ & 1.18 & 0.305 & \\
\hline 7 & Bronchitis & 54 & 19 & $35.2 \%$ & 2.72 & 0.001 & $* *$ & 12 & $22.2 \%$ & 1.60 & 0.167 & \\
\hline 11 & Hepatitis & 32 & 10 & $31.3 \%$ & 1.05 & 0.906 & & 3 & $9.4 \%$ & 0.67 & 0.515 & \\
\hline 12 & Gastric ulcer & 349 & 68 & $19.5 \%$ & 0.85 & 0.267 & & 49 & $14.0 \%$ & 0.90 & 0.533 & \\
\hline 13 & Gallstone & 144 & 45 & $31.3 \%$ & 1.88 & 0.001 & $* *$ & 19 & $13.2 \%$ & 0.72 & 0.214 & \\
\hline \multicolumn{13}{|c|}{$\begin{array}{l}\text { Are you currently taking any medicines } \\
\text { prescribed by your doctor? }\end{array}$} \\
\hline 1 & Yes & 4349 & 642 & $14.8 \%$ & 0.53 & 0.000 & $* * *$ & 653 & $15.0 \%$ & 2.11 & 0.000 & $* * *$ \\
\hline 2 & No & 1702 & 439 & $25.8 \%$ & 1.89 & 0.000 & $* * *$ & 180 & $10.6 \%$ & 0.46 & 0.000 & $* * *$ \\
\hline \multicolumn{13}{|c|}{ What medicine do you take? } \\
\hline 1 & Diabetes Mellitus & 155 & 67 & $43.2 \%$ & 2.86 & 0.000 & $* * *$ & 17 & $11.0 \%$ & 0.69 & 0.157 & \\
\hline
\end{tabular}

Table 2: Past medical history, Medicine use of participants.

number of brown rice eaters received regular health checkups, which might be related to that they had high health feeling, so not felt the necessity to go to the annual health checkup.

The physical activity level of the obese people was low compared to the non-obese people, and most of their work was sedentary and used a car when they moved. On the other hand, many brown rice eaters walked about 7,000 to 8,000 steps every day, and often did heavy work and intense exercise. Brown rice eaters slept 6-8 hours, waked up in good feeling in the morning, and refreshed (Table 3). As healthy eating habits, brown rice eaters often paid attention to select vegetable foods, low salt foods, small meals, and vegetarian foods. Even if obese people had health-consciousness, such as eating from vegetables and taking a low-carbohydrate diet, but many obese people preferred to eat oily foods, rich seasonings, oily, food, and people were eating until they were full (Table 3). These tended to be opposite for brown rice eaters.

As for supplements, many brown rice eaters decided to use it, and they took multi-mineral, vitamin B, vitamin C, iron, DHA and EPA, enzyme drink, fermented rice bran, amino acid/peptide, and 


\begin{tabular}{|c|c|c|c|c|c|c|c|c|c|c|c|c|}
\hline & & \multicolumn{6}{|c|}{ Obesity Group } & \multicolumn{5}{|c|}{ Brown rice eater Group } \\
\hline & & Total & Answer & $\%$ & OR & $\mathbf{p}$ & & Answer & $\%$ & OR & $\mathbf{p}$ & \\
\hline \multicolumn{13}{|c|}{ Select your physical activity level } \\
\hline 1 & $\begin{array}{l}\text { Mostly sitting work, moving mainly } \\
\text { by car }\end{array}$ & 2200 & 474 & $21.5 \%$ & 1.22 & 0.007 & $* *$ & 199 & $9.0 \%$ & 0.71 & 0.000 & $* * *$ \\
\hline 2 & Indoor work, housework, outing & 2689 & 394 & $14.7 \%$ & 0.92 & 0.273 & & 454 & $16.9 \%$ & 1.09 & 0.312 & \\
\hline 3 & Walk 7,000 to 8,000 steps every day & 789 & 146 & $18.5 \%$ & 0.87 & 0.197 & & 135 & $17.1 \%$ & 1.34 & 0.009 & ** \\
\hline 4 & $\begin{array}{l}\text { Doing hard work or strenuous exer- } \\
\text { cise }\end{array}$ & 437 & 82 & $18.8 \%$ & 0.89 & 0.388 & & 72 & $16.5 \%$ & 1.65 & 0.001 & $* * *$ \\
\hline \multicolumn{13}{|c|}{ Do you wake up in the morning? } \\
\hline 1 & I can't get up easily & 1170 & 166 & $14.2 \%$ & 0.93 & 0.456 & & 121 & $10.3 \%$ & 0.80 & 0.052 & \\
\hline 2 & Tiredness & 2071 & 424 & $20.5 \%$ & 1.28 & 0.001 & $* * *$ & 189 & $9.1 \%$ & 0.65 & 0.000 & $* * *$ \\
\hline 3 & Yes & 2831 & 493 & $17.4 \%$ & 0.82 & 0.006 & $* *$ & 531 & $18.8 \%$ & 1.69 & 0.000 & *** \\
\hline \multicolumn{13}{|c|}{ What do you practice as a diet? } \\
\hline 1 & Eat from vegetables & 2402 & 446 & $18.6 \%$ & 1.42 & 0.000 & $* * *$ & 335 & $13.9 \%$ & 1.07 & 0.374 & \\
\hline 2 & $\begin{array}{l}\text { Take } 5 \text { or more servings of vegetables } \\
\text { and } 200 \mathrm{~g} \text { of fruit daily }\end{array}$ & 95 & 15 & $15.8 \%$ & 1.10 & 0.738 & & 22 & $23.2 \%$ & 1.26 & 0.367 & \\
\hline 3 & Take 350g of vegetables daily & 239 & 33 & $13.8 \%$ & 0.89 & 0.563 & & 68 & $28.5 \%$ & 2.09 & 0.000 & $* * *$ \\
\hline 4 & Do not eat full & 459 & 44 & $9.6 \%$ & 0.46 & 0.000 & $* * *$ & 102 & $22.2 \%$ & 1.87 & 0.000 & $* * *$ \\
\hline 5 & Vegetable diet & 533 & 47 & $8.8 \%$ & 0.49 & 0.000 & $* * *$ & 232 & $43.5 \%$ & 5.52 & 0.000 & $* * *$ \\
\hline \multicolumn{13}{|c|}{ Do you usually eat to full stomach? } \\
\hline 1 & Eat to full stomach & 2635 & 558 & $21.2 \%$ & 1.72 & 0.000 & $* * *$ & 318 & $12.1 \%$ & 1.00 & 0.989 & \\
\hline 2 & Do not overeat & 3186 & 447 & $14.0 \%$ & 0.62 & 0.000 & $* * *$ & 416 & $13.1 \%$ & 0.95 & 0.505 & \\
\hline 3 & Eat about half & 187 & 19 & $10.2 \%$ & 0.54 & 0.014 & $*$ & 28 & $15.0 \%$ & 1.08 & 0.711 & \\
\hline \multicolumn{13}{|c|}{$\begin{array}{l}\text { Please choose the closest food prefer- } \\
\text { ence }\end{array}$} \\
\hline \multicolumn{13}{|c|}{ Oily food } \\
\hline 1 & Hardly eat & 1029 & 93 & $9.0 \%$ & 0.50 & 0.000 & $* * *$ & 310 & $30.1 \%$ & 3.42 & 0.000 & $* * *$ \\
\hline 2 & Modest & 3961 & 659 & $16.6 \%$ & 0.90 & 0.139 & & 383 & $9.7 \%$ & 0.44 & 0.000 & $* * *$ \\
\hline 3 & Eat willingly & 1039 & 282 & $27.1 \%$ & 1.96 & 0.000 & $* * *$ & 67 & $6.4 \%$ & 0.62 & 0.000 & $* * *$ \\
\hline \multicolumn{13}{|c|}{ Oily meat food (fatty meat, chicken skin) } \\
\hline 1 & Hardly eat & 2519 & 287 & $11.4 \%$ & 0.59 & 0.000 & $* * *$ & 533 & $21.2 \%$ & 2.78 & 0.000 & $* * *$ \\
\hline 2 & Modest & 2916 & 576 & $19.8 \%$ & 1.21 & 0.009 & ** & 213 & $7.3 \%$ & 0.43 & 0.000 & *** \\
\hline 3 & Eat willingly & 636 & 173 & $27.2 \%$ & 1.75 & 0.000 & $* * *$ & 32 & $5.0 \%$ & 0.51 & 0.000 & $* * *$ \\
\hline \multicolumn{13}{|c|}{ Supplements } \\
\hline 1 & Not Use & 4191 & 745 & $17.8 \%$ & 1.08 & 0.353 & & 388 & $9.3 \%$ & 0.46 & 0.000 & $* * *$ \\
\hline 2 & Use & 1754 & 273 & $15.6 \%$ & 0.94 & 0.476 & & 353 & $20.1 \%$ & 1.96 & 0.000 & $* * *$ \\
\hline \multicolumn{13}{|c|}{ What kind of supplements do you use? } \\
\hline 1 & Multi-vitamin & 383 & 64 & $16.7 \%$ & 0.99 & 0.968 & & 56 & $14.6 \%$ & 1.20 & 0.234 & \\
\hline 2 & Multi mineral & 190 & 32 & $16.8 \%$ & 1.00 & 0.994 & & 41 & $21.6 \%$ & 1.81 & 0.001 & ** \\
\hline 3 & Vitamin B & 240 & 36 & $15.0 \%$ & 0.93 & 0.706 & & 52 & $21.7 \%$ & 1.78 & 0.001 & $* * *$ \\
\hline
\end{tabular}




\begin{tabular}{|c|c|c|c|c|c|c|c|c|c|c|c|c|}
\hline 4 & Vitamin C & 377 & 41 & $10.9 \%$ & 0.69 & 0.035 & $*$ & 84 & $22.3 \%$ & 1.71 & 0.000 & *** \\
\hline 5 & Vitamin E & 176 & 21 & $11.9 \%$ & 0.76 & 0.254 & & 27 & $15.3 \%$ & 0.91 & 0.672 & \\
\hline 6 & Iron & 235 & 23 & $9.8 \%$ & 0.68 & 0.090 & & 46 & $19.6 \%$ & 1.63 & 0.005 & $* *$ \\
\hline 7 & Calcium & 209 & 30 & $14.4 \%$ & 0.97 & 0.897 & & 40 & $19.1 \%$ & 1.16 & 0.433 & \\
\hline 8 & DHA - EPA & 197 & 50 & $25.4 \%$ & 1.64 & 0.004 & $* *$ & 46 & $23.4 \%$ & 1.84 & 0.001 & $* * *$ \\
\hline 9 & Energy drink & 169 & 28 & $16.6 \%$ & 0.93 & 0.729 & & 21 & $12.4 \%$ & 0.93 & 0.771 & \\
\hline 10 & Enzyme drink & 97 & 9 & $9.3 \%$ & 0.58 & 0.132 & & 46 & $47.4 \%$ & 5.59 & 0.000 & $* *$ \\
\hline 11 & Fermented rice bran & 133 & 23 & $17.3 \%$ & 0.88 & 0.596 & & 51 & $38.3 \%$ & 4.70 & 0.000 & $* *$ \\
\hline 12 & Dietary fiber & 196 & 36 & $18.4 \%$ & 1.31 & 0.165 & & 31 & $15.8 \%$ & 1.02 & 0.921 & \\
\hline 13 & Oligosaccharide & 74 & 19 & $25.7 \%$ & 2.26 & 0.004 & $* *$ & 14 & $18.9 \%$ & 1.15 & 0.640 & \\
\hline 14 & Chondroitin sulfate & 61 & 14 & $23.0 \%$ & 1.78 & 0.069 & & 9 & $14.8 \%$ & 0.68 & 0.291 & \\
\hline 15 & Amino acid/Peptide & 99 & 16 & $16.2 \%$ & 0.95 & 0.864 & & 23 & $23.2 \%$ & 1.64 & 0.049 & $*$ \\
\hline 16 & Probiotics & 316 & 67 & $21.2 \%$ & 1.49 & 0.007 & $* *$ & 58 & $18.4 \%$ & 1.24 & 0.168 & \\
\hline 17 & Ginkgo leaf extract & 23 & 5 & $21.7 \%$ & 1.31 & 0.600 & & 9 & $39.1 \%$ & 3.52 & 0.005 & $* *$ \\
\hline
\end{tabular}

Table 3: Activity and sleep, diet, eating habits, preferences of participants.

ginkgo leaves. The educational background was higher in brown rice eaters, with less than $10 \%$ being middle and high school graduates.

Regarding individual food intake, brown rice eaters and obese people showed the remarkable contrast (Table 4).

Brown rice eaters seldom ate bread and cereals, but buckwheat was taken. Odds ratio of Azuki bean intake was very high (12.33). They took green yellow vegetables, pumpkin and carrot, and mushroom (shiitake), chestnut and sesame, but the intake of chicken, beef, pork, ham and bacon was significantly low. Soy bean protein, like natto and tofu substituted to animal protein. They did not take sugar and drank persimmon leaves tea. Obese people showed the reverse trends. They consumed beef, ham, bacon sausage, chicken and pork, and preferred to take soft drink, while green yellow vegetables and carrot intake was low compared to the non-obese people.

\section{Discussion}

Many epidemiology studies reported that high whole grain intake was associated with low risk of all-cause, cardiovascular, and cancer mortality [13-18]. Type 2 diabetes increased proportionally with BMI, and these could connect with other dietary and lifestyle factors $[19,20]$. It is often said that dietary restrictions and exercise were used to reduce the body weight of obesity, but it was difficult to succeed [7]. We confirmed the health condition of obese people were related to their past medical history and current use of medicine $[11,12]$. In particular, drugs for lifestyle related diseases, such as diabetes, hypertension, angina, hyperlipidemia and hyperuricemia were administered at an odds ratio of 2 - 3 among obese people. The fact that the risk of obesity was clearly shown by the odds ratio with respect to non-obese people suggests the necessity to prevent lifestyle related diseases by improving obesity.

The obesity people have characteristics in their dietary habits, such as preferring meat, no vegetables, oily foods, and eating until they became full. They had mostly sedentary occupation and moved mainly by car, and they were difficult to wake up in the morning, had a stressful lifestyle. Brown rice eaters consumed more healthy foods such as soy products, vegetable and sea-weeds, which include rich fiber, than those of white rice eaters. Therefore, the obese people need to voluntarily improve their lifestyle and change their behavior.

As we succeeded in the SCOP (SAKU Control Obesity Program) $[5,8]$, it was shown that obesity cannot be eliminated only by restricting calorie intake, and it was necessary to make behavioral change by existential therapeutic approach. The measures against 


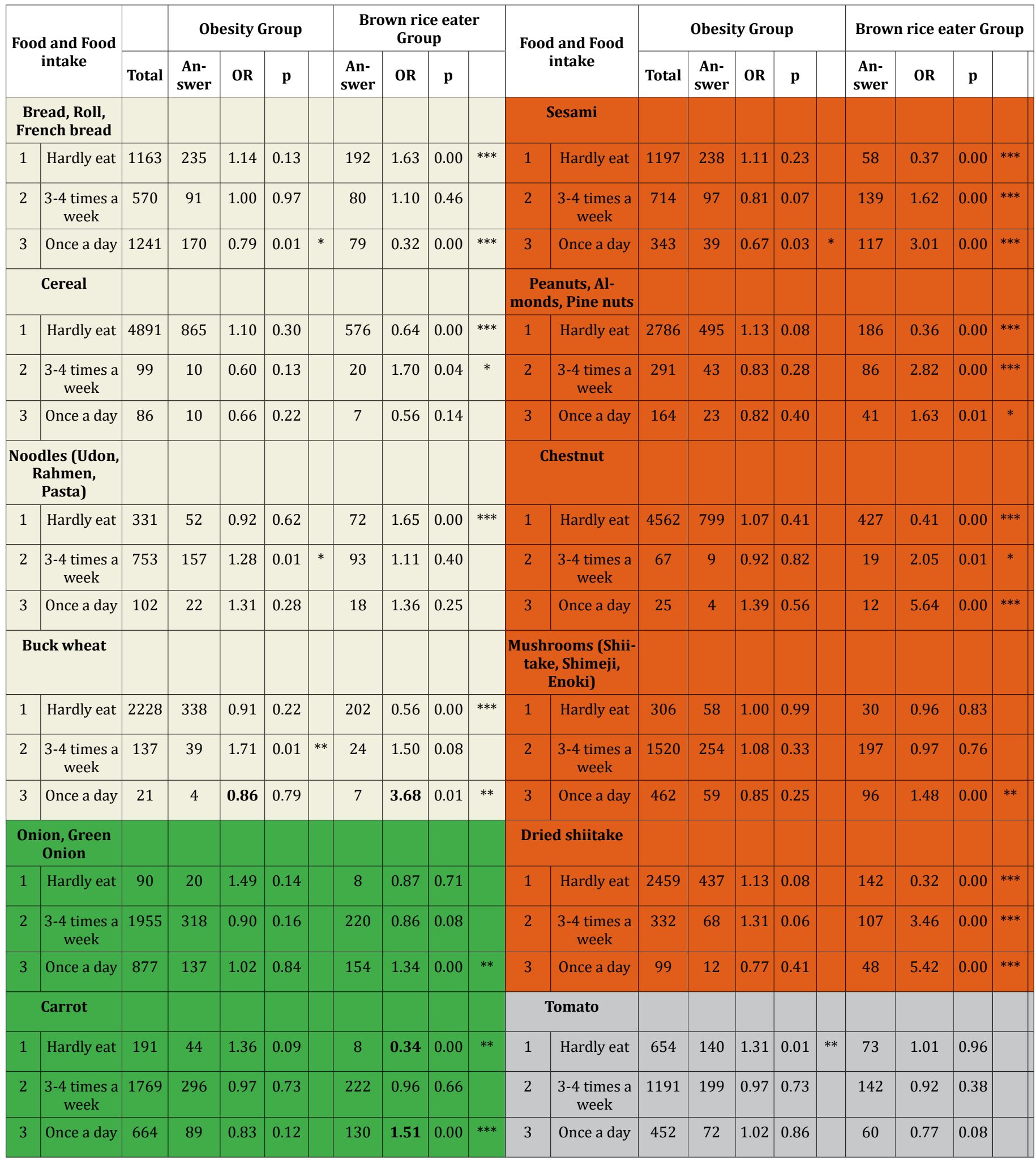




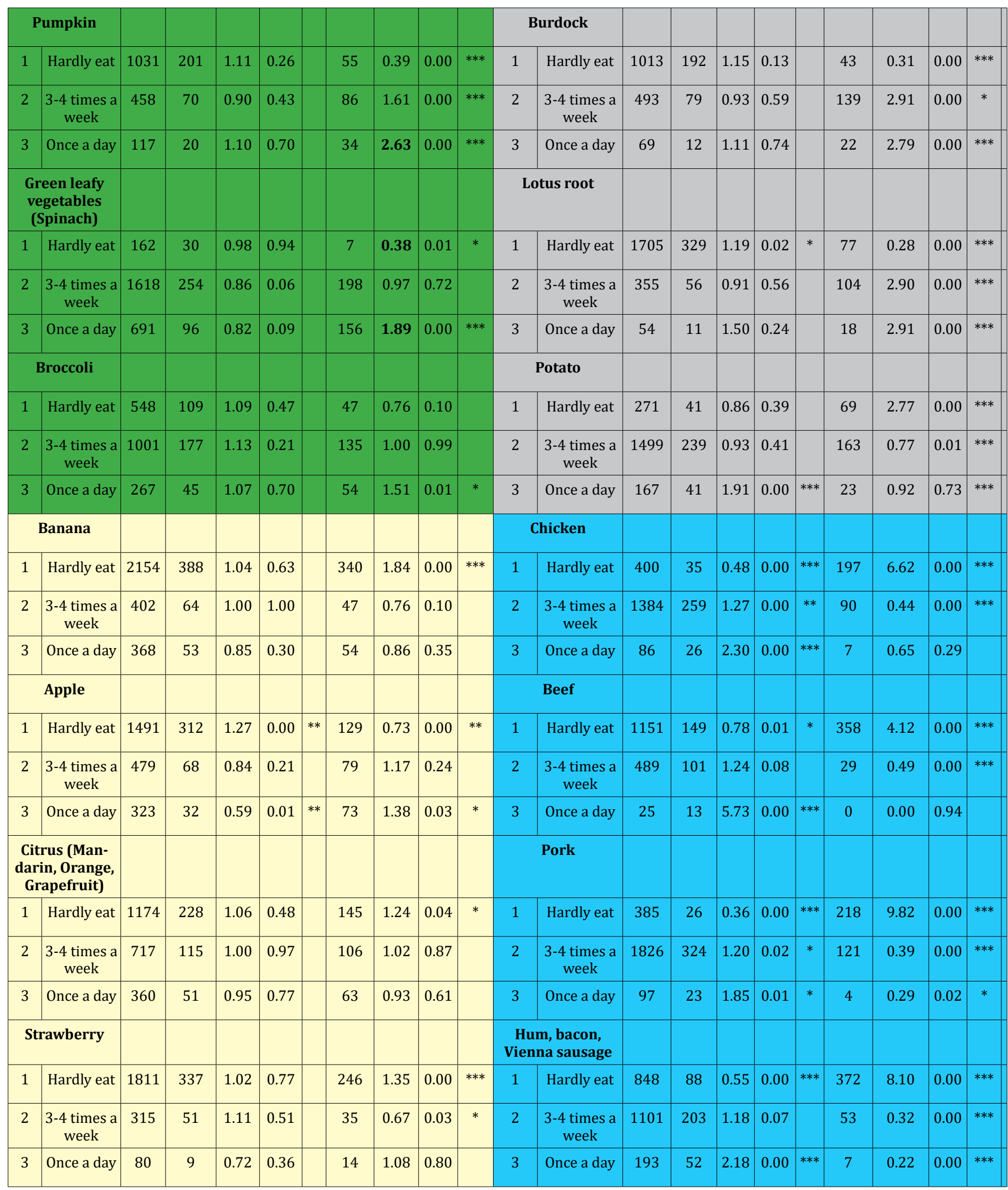




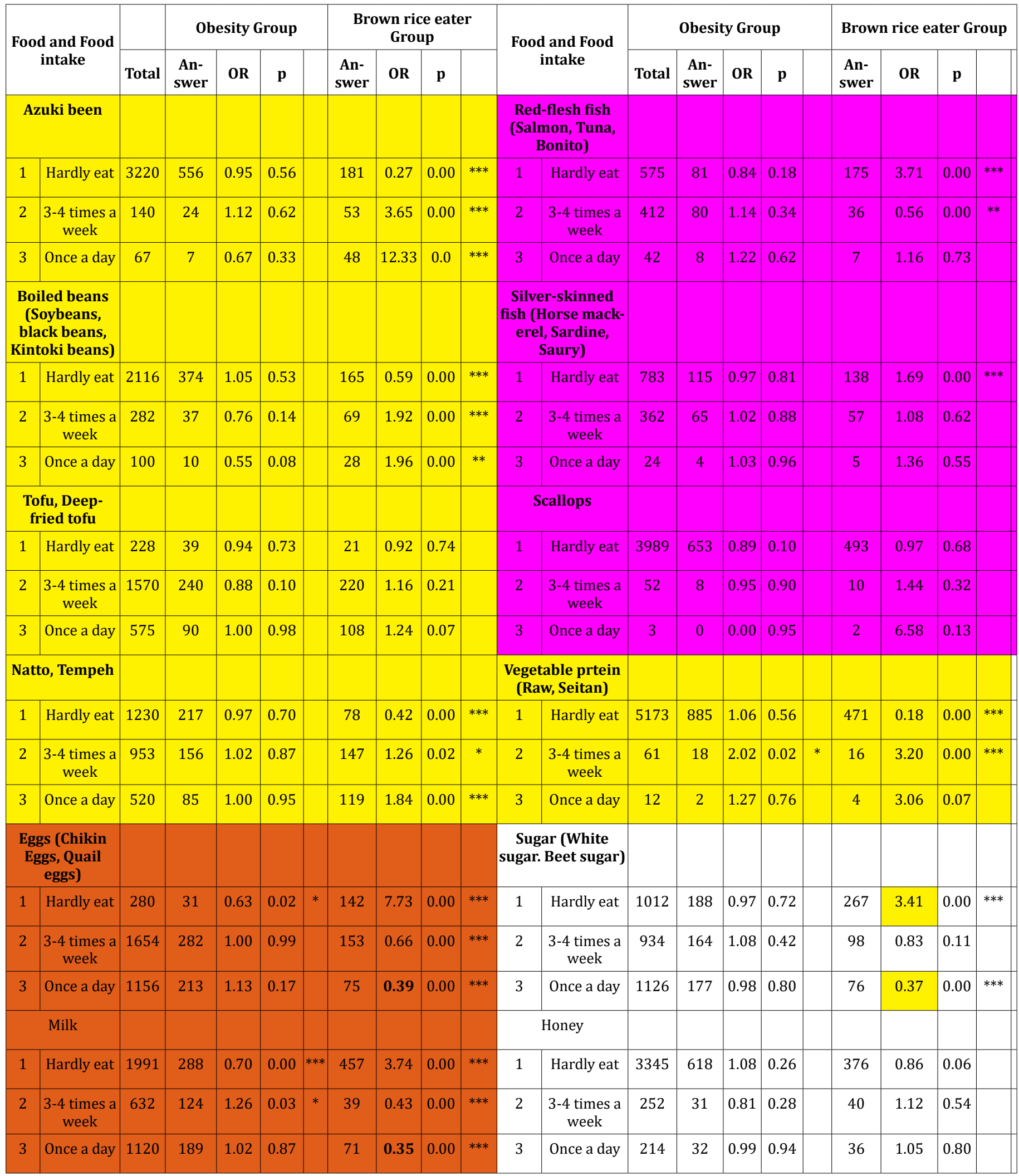




\begin{tabular}{|c|c|c|c|c|c|c|c|c|c|c|c|c|c|c|c|c|c|c|c|c|c|}
\hline \multicolumn{2}{|c|}{$\begin{array}{l}\text { Yoghurt, Fer- } \\
\text { mented milk } \\
\text { drink }\end{array}$} & & & & & & & & & & \multicolumn{2}{|c|}{ Green tea } & \multirow[b]{2}{*}{916} & \multirow[b]{2}{*}{118} & \multirow[b]{2}{*}{0.67} & \multirow[b]{2}{*}{0.00} & \multirow[b]{2}{*}{ *** } & \multirow[b]{2}{*}{223} & \multirow[b]{2}{*}{2.92} & \multirow[b]{2}{*}{0.00} & \multirow[b]{2}{*}{$* * *$} \\
\hline 1 & Hardly eat & 1322 & 212 & 0.77 & 0.00 & ** & 326 & 3.58 & 0.00 & *** & 1 & Hardly eat & & & & & & & & & \\
\hline 2 & $\begin{array}{c}3-4 \text { times a } \\
\text { week }\end{array}$ & 772 & 127 & 1.03 & 0.77 & & 68 & 0.61 & 0.00 & $* * *$ & 2 & $\begin{array}{c}\text { 3-4 times a } \\
\text { week }\end{array}$ & 603 & 117 & 1.19 & 0.12 & & 71 & 1.00 & 0.99 & \\
\hline 3 & Once a day & 1294 & 189 & 0.89 & 0.21 & & 109 & 0.43 & 0.00 & $* * *$ & 3 & Once a day & 948 & 181 & 1.12 & 0.22 & & 91 & 0.65 & 0.00 & $* * *$ \\
\hline \multicolumn{2}{|r|}{ Cheese } & & & & & & & & & & \multicolumn{2}{|c|}{$\begin{array}{c}\text { Persimmon leaves } \\
\text { tea }\end{array}$} & & & & & & & & & \\
\hline 1 & Hardly eat & 1448 & 256 & 0.96 & 0.59 & & 289 & 2.12 & 0.00 & $* * *$ & 1 & Hardly eat & 5797 & 995 & 1.15 & 0.43 & & 663 & 0.22 & 0.00 & $* * *$ \\
\hline 2 & $\begin{array}{c}\text { 3-4 times a } \\
\text { week }\end{array}$ & 701 & 126 & 1.21 & 0.08 & & 80 & 0.86 & 0.22 & & 2 & $\begin{array}{c}\text { 3-4 times a } \\
\text { week }\end{array}$ & 21 & 3 & 0.83 & 0.77 & & 9 & 6.17 & 0.00 & *** \\
\hline 3 & Once a day & 260 & 29 & 0.70 & 0.08 & & 20 & 0.43 & 0.00 & $* * *$ & 3 & Once a day & 14 & 3 & 1.85 & 0.36 & & 9 & 7.75 & 0.00 & *** \\
\hline \multicolumn{2}{|r|}{ Soymilk } & & & & & & & & & & \multicolumn{2}{|c|}{$\begin{array}{c}\text { Soft drink (Juice, } \\
\text { Carbonated } \\
\text { drink) }\end{array}$} & & & & & & & & & \\
\hline 1 & Hardly eat & 4023 & 736 & 1.09 & 0.24 & & 260 & 0.22 & 0.00 & *** & 1 & Hardly eat & 2894 & 354 & 0.55 & 0.00 & *** & 566 & 2.64 & 0.00 & * \\
\hline 2 & $\begin{array}{c}\text { 3-4 times a } \\
\text { week }\end{array}$ & 232 & 29 & 0.82 & 0.34 & & 64 & 2.65 & 0.00 & $* * *$ & 2 & $\begin{array}{l}\text { 3-4 times a } \\
\text { week }\end{array}$ & 482 & 119 & 1.57 & 0.00 & *** & 24 & 0.46 & 0.00 & \\
\hline 3 & Once a day & 262 & 33 & 0.84 & 0.37 & & 88 & 3.00 & 0.00 & $* * *$ & 3 & Once a day & 190 & 54 & 1.55 & 0.01 & $*$ & 13 & 0.67 & 0.17 & $* * *$ \\
\hline \multicolumn{2}{|c|}{ Wakame, Hijiki } & & & & & & & & & & \multicolumn{2}{|r|}{ Wine } & & & & & & & & & \\
\hline 1 & Hardly eat & 400 & 85 & 1.17 & 0.22 & & 20 & 0.41 & 0.00 & $* * *$ & 1 & Hardly eat & 4362 & 755 & 1.17 & 0.05 & & 473 & 0.54 & 0.00 & $*$ \\
\hline 2 & $\begin{array}{c}3-4 \text { times a } \\
\text { week }\end{array}$ & 1122 & 183 & 1.00 & 0.97 & & 184 & 1.38 & 0.00 & $* * *$ & 2 & $\begin{array}{l}\text { 3-4 times a } \\
\text { week }\end{array}$ & 134 & 18 & 0.71 & 0.19 & & 30 & 2.13 & 0.00 & \\
\hline 3 & Once a day & 326 & 43 & 0.86 & 0.37 & & 92 & 2.25 & 0.00 & $* * *$ & 3 & Once a day & 76 & 6 & 0.38 & 0.02 & * & 17 & 1.78 & 0.04 & \\
\hline \multicolumn{2}{|c|}{$\begin{array}{l}\text { Mozuku, Me- } \\
\text { kabu }\end{array}$} & & & & & & & & & & \multicolumn{2}{|c|}{ Chocolate, Cookie } & & & & & & & & & \\
\hline 1 & Hardly eat & 2523 & 424 & 0.97 & 0.71 & & 220 & 0.59 & 0.00 & $* * *$ & 1 & Hardly eat & 896 & 165 & 0.86 & 0.13 & & 182 & 2.18 & 0.00 & \\
\hline 2 & $\begin{array}{c}\text { 3-4 times a } \\
\text { week }\end{array}$ & 278 & 53 & 1.29 & 0.11 & & 65 & 1.80 & 0.00 & $* * *$ & 2 & $\begin{array}{l}\text { 3-4 times a } \\
\text { week }\end{array}$ & 1480 & 300 & 1.14 & 0.09 & & 202 & 1.17 & 0.09 & \\
\hline 3 & Once a day & 62 & 13 & 1.53 & 0.19 & & 22 & 2.59 & 0.00 & $* * *$ & 3 & Once a day & 391 & 56 & 1.05 & 0.75 & & 34 & 0.54 & 0.00 & $* * *$ \\
\hline \multicolumn{2}{|c|}{ Mayonnaise } & & & & & & & & & & \multicolumn{2}{|c|}{$\begin{array}{l}\text { Cake (Cake, Cas- } \\
\text { tilla, Cream puff) }\end{array}$} & & & & & & & & & \\
\hline 1 & Hardly eat & 935 & 128 & 0.76 & 0.01 & $* *$ & 268 & 3.79 & 0.00 & $* * *$ & 1 & Hardly eat & 1620 & 275 & 0.80 & 0.01 & $* *$ & 302 & 2.14 & 0.00 & $*$ \\
\hline 2 & $\begin{array}{c}\text { 3-4 times a } \\
\text { week }\end{array}$ & 1216 & 228 & 1.49 & 0.10 & & 92 & 0.51 & 0.00 & $* * *$ & 2 & $\begin{array}{l}\text { 3-4 times a } \\
\text { week }\end{array}$ & 203 & 36 & 1.27 & 0.22 & & 20 & 0.75 & 0.24 & \\
\hline 3 & Once a day & 252 & 49 & 1.20 & 0.28 & & 17 & 0.45 & 0.00 & $* *$ & 3 & Once a day & 25 & 4 & 1.41 & 0.54 & & 2 & 0.49 & 0.34 & $* * *$ \\
\hline \multicolumn{2}{|r|}{ Herb tea } & & & & & & & & & & \multicolumn{2}{|c|}{ Ice cream } & & & & & & & & & \\
\hline 1 & Hardly eat & 4632 & 843 & 1.14 & 0.16 & & 444 & 0.38 & 0.00 & $* * *$ & 1 & Hardly eat & 2039 & 285 & 0.65 & 0.00 & $* * *$ & 397 & 2.11 & 0.00 & \\
\hline 2 & $\begin{array}{c}\text { 3-4 times a } \\
\text { week }\end{array}$ & 132 & 19 & 1.12 & 0.66 & & 31 & 2.01 & 0.00 & $* *$ & 2 & $\begin{array}{l}\text { 3-4 times a } \\
\text { week }\end{array}$ & 309 & 61 & 1.30 & 0.08 & & 34 & 0.95 & 0.78 & \\
\hline 3 & Once a day & 81 & 10 & 0.80 & 0.52 & & 25 & 2.68 & 0.00 & $* * *$ & 3 & Once a day & 63 & 15 & 1.51 & 0.17 & & 3 & 0.29 & 0.04 & $* * *$ \\
\hline
\end{tabular}

Table 4: Food intake in obese and brown rice diets. 
metabolic syndrome have been accepted by "Healthy Japan 21", but we are considering that there is not enough for psychological support as a part of the individual support.

In contrast to the obese people, the brown rice eaters were in excellent health condition and almost no obese. There was fewer past medical history, and odds ratios for drug use such as for hypertension, hyperlipidemia, and angina were 0.2 - 0.3 [11,12]. In the changes of healthy feeling from the last year, OR of "Better" was 3.74 among brown rice eaters, which contrasted with OR of 0.6 for obese people. They seemed not to want to eat meat or to go for a health checkup because brown rice eaters had healthy feeling by getting enough nutrition from brown rice. Recently functional ingredients of brown rice became the evidence of recommendation [21-25].

Many brown rice eaters have a high educational background, and it would be a reason why the knowledge about the nutrition of brown rice was linked to the choice of brown rice. Many brown rice eaters took supplements, so it was also a reflection of their health consciousness.

The food intake and dietary habits have many implications for the post corona society and future aging society [26]. Besides, grain consumption's ratio in carbohydrates has been reduced during the past 40 years in Japan. Especially when dealing with the percentage of rice consumption, "rice and rice products" has become under $50 \%$.

Much more intervention studies are needed to accumulate enough evidence to recommend consumption of brown rice of whole grain or other types of processed rice (we call these kind of rice as "Medical Rice"). This is a new concept of the rice which has the functional effect for disease prevention and dietary therapy as "Medical Rice" [27-29].

For implementing the strategy of "Rice change" behavior as the "supportive social environment" approach, we consider to more effective developing partnership among consumer, food processor, and producer. So, Watanabe., et al. has constructed "Medical Rice Association" in 2019. The association's members joined from diversified areas and their backgrounds were medical specialists, nutritionists, agricultural members $[27,28]$.
As the recommendation strategy of variety foods intake for aged people, changing staple food to brown rice of whole grain may associate with increasing healthy side dish food intake. From two large-scale epidemiologic studies conducted in Greece [16], higher intake of whole grains evaluated by mainly "whole wheat" of bread was associated with a higher level of "successful aging" index. These were evaluated by 10 attributes such as health-related, social lifestyle and clinical factors.

We recently found that rice eating countries showed the less incidence and mortality of COVID-19 [24].

There are many additional benefit in brown rice eating.

WHO had proposed the importance of food based guideline. Our proposal is coincided with the WHO recommendation. Relationship of intestinal microbiota and health further support the importance of Genmai eating. Health related factors of rice should be a possible future research topic. Therefore, much more scientific evidence for healthy rice is needed for implementation of "dietary education" and "supportive environment" for healthy people and preliminary one.

\section{Acknowledgment}

The authors appreciate the participants to the GENKI Study 1 and 2 , and many collaborative staffs.

\section{Conflict of Interest}

The authors had no declaration of COI.

\section{Funding}

The research was supported by the Llifescience Promoting Association and a part of this work was supported by the Ministry of Agriculture, Forestry and Fisheries and the grant from the Project of the NARO Bio-oriented Technology Research Advancement Institution. (Research program on development of innovative technology).

\section{Bibliography}

1. WHO expert consultation. "Appropriate body mass index for Asian populations and its implications for policy and intervention strategies". Lancet 363 (2004): 157-163.

2. Ministry of Health, Labour and Welfare. Diabetes Mellitus (2019). 
3. Sasaki S., et al. "Food and nutrient intakes assessed with dietary records for the validation study of a self-administered food frequency questionnaire in JPHC Study Cohort I". Journal of Epidemiology 13 (2003): 23-50.

4. Sasaki S., et al. "Validity of a self-administered food frequency questionnaire used in the 5-year follow up survey of the JPHC Study Cohort I to assess dietary fiber intake: comparison with dietary records". Journal of Epidemiology 13 (2003): S106114.

5. Watanabe S., et al. "Study design of the Saku Control Obesity Program (SCOP)". Anti-Aging Medicine 4.3 (2007): 70-73.

6. Tsugane S., et al. "Validity and reproducibility of the self-administered food frequency questionnaire in the JPHC Study Cohort I: study design, conduct and participant profiles". Journal of Epidemiology 13 (2003): 2-12.

7. Naade M., et al. "What behaviors are important for successful weight maintenance?" Journal of Obesity (2012): 202037.

8. Kawashima N., et al. "Changes of fat volume and adipocytokines by the randomized intervention program for obesity control program (SCOP)". Diabetes Research Open Journal 1.5 (2016): 136-146.

9. Ojo 0. "An overview of diabetes and its complications". Diabetes Research Open Journal 2 (2016): e4-e6.

10. Tanaka S., et al. "Cohort Profile: The Japan Diabetes Complications Study: a long-term follow-up of a randomized lifestyle intervention study of type 2 diabetes". International Journal of Epidemiology 43 (2014): 1054-1062.

11. Watanabe S., et al. "Effects of brown rice on obesity: GENKI Study I (Cross sectional epidemiological study". Journal of Obesity and Metabolic Disorder 2.1 (2018): 12-19.

12. Hirakawa A., et al. "The Nested Study on the Intestinal Microbiota in GENKI Study with Special Reference to the Effect of Brown Rice Eating". Journal of Obesity and Metabolic Disorder 3.1 (2019): 1-13.

13. Zhang B., et al. "Association of whole grain intake with, allcause, cardiovascular, and cancer mortality: a systematic review and, dose-response meta-analysis from prospective cohort studies". European Journal of Clinical Nutrition 72 (2017): $57-65$.
14. Mohan V., et al. "Effect of brown rice, white rice, and brown rice with legumes on blood glucose and insulin responses in overweight Asian Indians: a randomized controlled trial". Diabetes Technology and Therapeutics 16.5 (2014): 317-325.

15. Foscolou A., et al. "The association between whole grain products consumption and successful aging: A combined analysis of MEDIS and ATTICA Epidemiological Studies". Nutrients 11.6 (2019): 1221.

16. GBD 2017 Diet Collaborators. "Health effects of dietary risks in 195 countries, 1990-2017: a systematic analysis for the Global Burden of Disease Study". Lancet 393 (2019): 1958-1972.

17. Aune D., et al. "Whole grain consumption and risk of cardiovascular disease, cancer, and all cause and cause specific mortality: systematic review and dose-response meta-analysis of prospective studies". BMJ 353 (2016): i2716.

18. Aune D., et al. "Whole grain and refined grain consumption and the risk of type 2 diabetes: a systematic review and doseresponse meta-analysis of cohort studies". European Journal of Epidemiology 28 (2013): 845-858.

19. Zhang G., et al. "Substituting brown rice for white rice to lower diabetes risk: a focus-group study in Chinese adults". Journal of the American Dietetic Association 110.8 (2010): 1216-1221.

20. Maki KC., et al. "The Relationship between Whole Grain Intake and Body, Weight: Results of Meta-Analyses of Observational Studies and Randomized, Controlled Trials". Nutrients 11 (2019): pii: E1245.

21. Inoue M., et al. "Impact of body mass index on the risk of total cancer incidence and mortality among middle-aged Japanese: data from a large-scale population-based cohort study-the JPHC study". Cancer Causes Control 15.79 (2004): 671-680.

22. Singh RB., et al. "Medical rice as a whole grain: A new technological advancement by Shaw Watanabe, for prevention of cardiometabolic diseases and dementia". Biomedical Journal of Scientific and Technical Research 14.4 (2019): 10927-10831.

23. Ito Y., et al. "Postprandial blood glucose and insulin responses to pre-germinated brown rice in healthy subjects". The Journal of Medical Investigation 52 (2005): 159-164. 
24. Masuzaki H., et al. "Brown rice-specific $\gamma$-oryzanol as a promising prophylactic avenue to protect against diabetes mellitus and obesity in humans". Journal of Diabetes Investigation 10 (2019): 18-25.

25. Watanabe S., et al. "Medical and Nutraceuticals in Metabolic and Non-Communicable Diseases". Elsevier (in press) (2020).

26. Watanabe $S$ and Inuma K. "Low COVID-19 infection and mortality in rice eating countries". Scholar Journal of Food and $\mathrm{Nu}$ trition (2020).

27. Watanabe S., et al. "Food as medicine: The new concept of "medical rice". Advances in Food Technology and Nutritional Sciences 2.2 (2016): 38-50.

28. Van der Kamp JW., et al. "The HEALTHGRAIN, definition of 'whole grain". Food and Nutrition Research 58 (2014): 10.

29. Watanabe S. "Evidence of Brown Rice". KIRASIENNE Inc. Tokyo, Japan (2015).

\section{Assets from publication with us}

- Prompt Acknowledgement after receiving the article

- Thorough Double blinded peer review

- Rapid Publication

- Issue of Publication Certificate

- High visibility of your Published work

Website: www.actascientific.com/

Submit Article: www.actascientific.com/submission.php

Email us: editor@actascientific.com

Contact us: +919182824667 